\title{
A MULTI PARAMETRIC QUADRATIC PROGRAMMING SOLUTION TO ROBUST PREDICTIVE CONTROL
}

\author{
J.A. Rossiter ${ }^{* *}$ B. Pluymers* 1 J.A.K. Suykens * \\ B. De Moor* 2 \\ * Research Group SCD-SISTA, Department Of \\ Electrotechnical Engineering (ESAT), \\ Katholieke Universiteit Leuven, Kasteelpark Arenberg 10, \\ 3001 Leuven, Belgium \\ \{bert.pluymers, johan. suykens, bart. demoor\}@esat.kuleuven.ac.be \\ ** Department of Automatic Control and Systems \\ Engineering, Mappin Street, University of Sheffield, S1 \\ 3JD, $U K$ \\ j.a.rossiter@sheffield.ac.uk
}

\begin{abstract}
Multi parametric quadratic programming is an alternative means of implementing conventional predictive control algorithms whereby one transfers much of the computational load to offline calculations. This paper demonstrates how one can formulate a robust MPC problem as a quadratic program and hence make it amenable to MPQP solutions. The paper then derives some MPQP solutions and discusses the efficacy of these. Copyright ${ }^{\circledR} 2005$ IFAC
\end{abstract}

Keywords: Parametric programming, quadratic programming, robust MPC

\section{INTRODUCTION}

In recent years Multi parametric quadratic programming (MPQP) (Bemporad et al., 2002) has become a useful tool for characterising, offline, the model predictive control (MPC) algorithm. The effectiveness of this approach is due to several factors: (i) the online implementation reduces to a look up table and hence is easy to code and (ii) the structure of the control strategy is transparent, which is not the case when using an online optimisation. Naturally there are limitations and much of the current research is investigating ways of simplifying the computation and complexity of the solution as these are significant barriers to applications with many states.

This paper however is concerned with a different aspect, that is robustness. Much of the works in

\footnotetext{
1 B. Pluymers is a research assistant with the I.W.T. Flanders.

2 Research partially supported by Research Council KULeuven : GOA-Mefisto 666, FWO projects : G.0240.99, G.0197.02, G.0491.03, Belgian Federal Government : IUAP V-22 (2002-2006)
}

the literature (Bemporad et al., 2001; Borrelli et al., 2001; Rossiter, 2004; Tondel et al., 2003; Johansen et al., 2003) concentrate on the linear case (hybrid and continuous time systems are not the topic of this paper). Yet if one looks at the MPC literature outside of the topic of MPQP, much of the current emphasis is on either robustness issues e.g. (Kothare et al., 1996; Kouvaritakis et al., 2000; Kerrigan, 2000) or non-linear systems. What makes this dichotomy seem even more surprising is that the very power of MPQP is its ability to handle the heavy computations offline and it is the need for burdensome optimisations that has severely limited the progress of robust MPC towards implementable algorithms; in fact this is recognised in some of the current offerings (Kouvaritakis et al., 2000; Kothare et al., 2003) which sacrifice some optimality and feasibility in order to deliver a simpler implementation.

Hence this paper has the purpose of considering how one might use the potential of MPQP to implement a robust MPC law. The solution may seem to be relatively obvious, but the application to robust MPC has specific advantages in the 
sense that the number of MPQP regions is shown to rise less steeply than the number of constraints in the QP formulation and hence facilitates online implementation.

The main reason why MPQP is difficult to apply to robust systems is that one needs to capture a whole class of predictions as opposed to just the nominal. Although this may seem tractable for a few steps, the combinatorial explosion (Schuurmanns et al., 2000) of the possible predictions makes this intractable for even relatively low horizons and of course the usual thinking within MPC (Mayne et al., 2000; Rossiter, 2003 ) is that large horizons are to be prefered in general. Of course there are ways around this, for instance works concentrating on disturbance rejection (Kerrigan, 2000) make use of one-step sets and backwards prediction as such an approach avoids the combinatorial difficulties.

However, many implementations nevertheless tend to rely upon the definition of an invariant and feasible terminal region, that is a space in which the trajectories for all possible uncertainties will remain once they have entered and of course, satisfy constraints. The existence of such a target region (Scokaert et al., 1998; Rossiter et al., 1998) allows one to restrict the active prediction horizon, $n_{c}$, to the same length as the number of free control moves; usually a low number and hence manageable even with a combinatorial growth in possible trajectories. Unfortunately, in the case of uncertain systems, most commonly modelled as linear parameter varying (LPV) systems, authors have generally assumed that maximal volume invariant terminal regions are not computable easily and have pursued other avenues. Typical routes in the literature assume that the invariant regions are ellipsoidal (which implies that the MPC algorithm becomes a semi-definite program (Cannon et al., 2001)) or low complexity polyhedrals (Cannon et al., 2002) (which severely restricts the feasible region); moreover these works often assume symmetric constraints. However, the good news is that a recent work (Pluymers et al., 2004) demonstrated that, under certain conditions, it is possible to compute a maximal volume polyhedral terminal region for an LPV system; this result underpins the developments presented here.

This paper therefore unites several concepts:

(1) The use of a terminal region for robust MPC to keep the active prediction horizon small and hence to handle the combinatorial growth of possible trajectories.

(2) The use of backwards prediction in order to define the terminal region.

(3) Once the control law has been established as deploying a QP optimiser, it can immediately be solved as an MPQP.
(4) The method is not restricted to symmetric constraints (a typical assumption in many papers) and hence extends feasibility extensively.

The paper is organised as follows. Section 2 gives some background to the formulation of the robust MPC algorithm (Pluymers et al., 2004), section 3 dicusses the MPQP implementation for the robust and nominal algorithms and section 4 gives some numerical illustrations of the differences. Section 5 contains conclusions.

\section{BACKGROUND}

\subsection{A nominal MPC algorithm}

This paper will assume discrete LPV state space models

$$
\begin{gathered}
x_{k+1}=A(k) x_{k}+B(k) u_{k} \\
(A(k), B(k)) \in C o\left\{\left(A_{1}, B_{1}\right), \ldots,\left(A_{m}, B_{m}\right)\right\}
\end{gathered}
$$

with the nominal model being $(A, B)$. The 'predicted' control law (Rossiter et al., 1998; Scokaert et al., 1998) takes the form

$$
\begin{array}{ll}
u_{k}=-K x_{k}+c_{k} & k=0, \ldots, n_{c}-1 \\
u_{k}=-K x_{k} & k \geq n_{c}
\end{array}
$$

where only the first move of this is ever implemented; $c_{k}$ are d.o.f. available for constraint handling and thus are chosen as $c_{i}=0$ when this is feasible. Performance, either predicted or actual, will be assessed by the cost

$$
J=\sum_{k=0}^{\infty} x_{k}^{T} Q x_{k}+u_{k}^{T} R u_{k}
$$

Substituting a nominal model and 'optimal' control law (2) into (3) and ignoring terms that do not depend upon the d.o.f., one can show (Rossiter, 2003) that the cost to be minimised, $J_{c}$, takes the form

$$
J_{c}=C^{T} W_{D} C ; \quad C=\left[c_{0}^{T}, \ldots, c_{n_{c}-1}^{T}\right]^{T}
$$

where $W_{D}=\operatorname{diag}(W, \ldots, W)$, and $W=B^{T} \Sigma B+$ $R, \Sigma-\Phi^{T} \Sigma \Phi=Q+K^{T} R K$.

Assume that the process is subject to constraints:

$$
\underline{u} \leq u_{k} \leq \bar{u} ; \quad \underline{x} \leq x_{k} \leq \bar{x}, \quad k=0, \ldots, \infty
$$

Then the maximal admissible set $^{1}$ (MAS) $S_{0}$ (Gilbert et al., 1991) can be defined as:

$$
S_{0}=\left\{x: M_{0} x \leq d_{0}\right\}
$$

The maximal control admissible set (MCAS) $S_{c}$, the set from which in at most $n_{c}$ moves one can drive the state into $S_{0}$, again without violating constraints, takes the form:

$$
S_{c}=\left\{x: \exists C \text { s.t. } M_{0} x+N_{0} C \leq d_{0}\right\}
$$

\footnotetext{
1 The largest invariant set in which the state trajectories do not violate constraints.
} 
Finally therefore, a popular MPC algorithm with guarantees of recursive feasibility and convergence, for the nominal case, is given as:

MPC Algorithm: At each sampling instant, minimise the performance index:

$$
\min _{C} J=C^{T} W_{D} C \text { s.t. } M_{0} x+N_{0} C \leq d_{0}
$$

where only the first block element of $C$ is implemented.

\subsection{RMPC: $M P C$ for the $L P V$ case}

Before moving onto the MPQP implementation, it is next necessary to derive a robust MPC (RMPC) algorithm deploying just an QP optimisation. For ease of presentation, we summarise the result first and the remainder of this section gives more detail which the reader could choose to skip.

2.2.1. The key result An RMPC algorithm can be summarised by the optimisation:

RMPC algorithm: At each sampling instant, minimise the performance index:

$$
\min _{C} J=C^{T} W_{D} C \text { s.t. } M_{r} x_{k}+N_{r} C \leq d_{r}
$$

where only the first block element of $C$ is implemented.

It has been argued (Pluymers et al., 2004) that one can define inequalities such that the control law implied in (9) has:

(1) Recursive feasibility for system (1).

(2) Guaranteed stability and convergence for all initial points inside $S_{r}$ (which is the MCAS for RMPC),

$$
S_{r}=\left\{x: \exists C \text { s.t. } M_{r} x_{k}+N_{r} C \leq d_{r}\right\} .
$$

For completeness define the associated MAS as $S_{v}$, that is

$$
S_{v}=\left\{x: M_{r} x \leq d_{r}\right\}
$$

Remark 2.1. RMPC of (9) differs from MPC of (8) only in the inequalities that need to be satisfied, or in other words the replacement of $S_{0}$ by $S_{v}$ and $S_{c}$ by $S_{r}$. The key to the success of RMPC therefore is in the definition of these terms, that is, $M_{r}, N_{r}, d_{r}$.

2.2.2. Outline of algorithm development Next we give a brief overview of why algorithm (9) is robustly stabilising in the presence of constraints (5); for more details see (Pluymers et al., 2004).

Performance index: This is based on nominal performance but in particular is phrased in terms of perturbations to the nominal control law. If one knows that the nominal feedback is stabilising for the LPV system (1), then one can prove convergence by establishing that the perturbation term $C$ is decreasing. Given recursive feasibility, this can be determined from standard Lyapunov arguments.

Recursive feasibility: The key to (9) is the requirement that

$$
x_{k} \in S_{r} \quad \Rightarrow \quad x_{k+1} \in S_{r}
$$

and in fact to be more precise, one requires that an augmented state including the tail of $C$ remains feasible, that is:

$\left[M_{r}, N_{r}\right]\left[\begin{array}{c}x_{k} \\ C_{k}\end{array}\right] \leq d_{r} \Rightarrow\left[M_{r}, N_{r}\right]\left[\begin{array}{c}x_{k+1} \\ C_{t a i l \mid k}\end{array}\right] \leq d_{r}$

where $C_{t a i l \mid k}=\left[c_{k+1 \mid k}^{T}, \ldots, c_{k+n_{c}-1 \mid k}, 0\right]^{T}$. Such a guarantee can be established by defining $M_{r}, N_{r}, d_{r}$ as the combination of several constraints:

(1) constraint matrices that ensure constraint satisfaction for all possible state trajectories over $n_{c}$ steps.

(2) a constraint that ensures all possible $n_{c}$ step ahead predictions enter the MAS $S_{v}$.

The precise details of how to compute these matrices are omitted to save space and are available in (Pluymers et al., 2004).

\section{THE MPQP IMPLEMENTATION}

\subsection{The structure of an MPQP algorthm}

MPQP (Bemporad et al., 2002) gives a parameterisation of the solution to a $\mathrm{QP}$ in terms of the variables which affect the cost and constraints but are not degrees of freedom. In the case of $(8,9)$ this parameter is the state $x$. It is known that the optimum solution (in this case for $C$ ) has a piecewise affine (PWA) dependence on the state. So for instance, specific solutions in a given region take the form:

$x \in S_{i} \Rightarrow C=-K_{i} x+p_{i} ; \quad S_{i}=\left\{x: M_{i} x \leq d_{i}\right\}$

and the overal solution is defined over the union of all the regions $S_{i}$ (which do not overlap). Moreover it can be shown that this union is equivalent to the MCAS $S_{r}$, that is:

$$
S_{r}=\bigcup_{i} S_{i}
$$

Remark 3.1. One of the regions will be the MAS $\left(S_{v}\right)$; for this region $K_{i}=p_{i}=0$.

Remark 3.2. The definitions of $M_{i}, d_{i}, K_{i}, p_{i}$ depend in a non-simple way upon the parameters of $(8,9)$ the original optimisation (for instance $\left.W_{D}, M_{r}, N_{r}, d_{r}\right)$. As such one may find a relatively small change in the inequalities gives substantial changes to the shape and number of regions $S_{i}$ as well as the corresponding $K_{i}, p_{i}$. 


\subsection{Differences between the nominal and robust algorithms}

A quick glance is sufficient to note that optimisations (8) and (9) are of the same structure but differ in the inequalities. It is therefore expected that the solutions may look quite different. This in itself is very insightful because a simple plot can be used to demonstrate how the control law changes, with the state position, to accomodate the model uncertainty. Such plots are given in the next section.

Another interesting issue is complexity. The 'nominal' inequalities used in $(8,9)$ are very different in dimension (no. or rows) due to the combinatorial nature of the predictions for the robust case. Although some simplification maybe possible offline by removing redundant rows, it would still be unsurprising if the robust algorithm did not have many more rows and hence could potentially ${ }^{2}$ require significantly longer solution times for the online implementation. The hope, however, might be that the MPQP solution may not be substantially more complex. In this case one would be able to handle the robust problem with little more effort than the nominal case and that would be a significant benefit.

\section{NUMERICAL ILLUSTRATIONS}

The purpose of this section is simply to compute the MPQP (Kvasnica et al., 2003) solution for the robust MPC algorithm, as this in itself is novel and then to compare it to the nominal MPC algorithm. It is perhaps self evident that $\mathrm{RMPC}$ will be more robust, although this is also illustrated, so our prime interest is to contrast the complexity of the two solutions so that some comment can be made about the potential of MPQP solutions to RMPC.

For simplicity of illustration and to aid comparison with other publications, we use the two state, double integrator example used by many other authors. The model takes the form of (1) where

$$
\begin{array}{rlrl}
A_{1} & =\left[\begin{array}{cc}
1 & 0.1 \\
0 & 1
\end{array}\right], & B_{1}=\left[\begin{array}{l}
0 \\
1
\end{array}\right], \\
A_{2}=\left[\begin{array}{cc}
1 & 0.2 \\
0 & 1
\end{array}\right], & B_{2}=\left[\begin{array}{c}
0 \\
1.5
\end{array}\right],
\end{array}
$$

and $A(k)=(1-\lambda(k)) A_{1}+\lambda(k) A_{2}, \quad B(k)=$ $(1-\lambda(k)) B_{1}+\lambda(k) B_{2}, 0 \leq \lambda(k) \leq 1$. For the purposes of this section to ensure consistency of comparisons, $\lambda(k)$ is time-varying but defined to be the same sequence for all simulations. The nominal model is chosen as $A=0.5\left(A_{1}+A_{2}\right), B=$ $0.5\left(B_{1}+B_{2}\right)$. Constraints are taken as

$$
\bar{u}=1 ; \quad \underline{u}=-0.5 ; \quad \bar{x}=-\underline{x}=[10,10]^{T} .
$$

\footnotetext{
2 Although one could argue that in general the number of rows is less significant a factor than the number of d.o.f..
}

The unconstrained feedback controller $K=$ $\left[\begin{array}{ll}0.4558 & 0.3698\end{array}\right]^{\mathrm{T}}$ is the LQR-optimal for $Q=$ $\operatorname{diag}(1,0.01), R=3$.

\subsection{Number of constraints vs number of d.o.f.}

We first illustrate how the complexity of the underlying optimisations (8) and (9) differ by presenting, in table 1 , the number of inequalities required to define $S_{c}, S_{r}$ for various $n_{c}$. Unsurprisingly, RMPC requires significantly more inequalities, because it is capturing all possible predictions, not just the nominal. This could cause a marked slow down in online implementation times of the associated QP optimisation.

\begin{tabular}{|l|c|c|}
\cline { 2 - 3 } \multicolumn{1}{c|}{} & \multicolumn{2}{|c|}{ Number of inequalities } \\
\hline$n_{c}$ & MPC & RMPC \\
\hline 1 & 13 & 17 \\
2 & 15 & 31 \\
3 & 19 & 56 \\
4 & 21 & 102 \\
5 & 24 & 194 \\
\hline
\end{tabular}

Table 1. Comparison of inequalities

\subsection{MPQP complexity comparisons}

Next we consider, in table 2, the complexity of the MPQP solutions, that is how many regions are required to cover the MCAS. It is clear that the complexity of the MPC solution is less than for RMPC and moreover there seems to be a trend that the the number of regions for RMPC increases more rapidly with $n_{c}$. Nevertheless, when compared to the number of inequalities (table 1) the MPQP solution of RMPC relative to MPC could still be argued to be more favourable than the $\mathrm{QP}$ comparison; for instance at $n_{c}=5$ the relative number of regions is approximately 3 whereas the relative number of inequalities is approximately 7.5 .

\begin{tabular}{|l|c|c|}
\cline { 2 - 3 } \multicolumn{1}{c|}{} & \multicolumn{2}{|c|}{ Number of regions } \\
\hline$n_{c}$ & MPC & RMPC \\
\hline 1 & 14 & 16 \\
2 & 42 & 68 \\
3 & 73 & 124 \\
4 & 102 & 204 \\
5 & 131 & 313 \\
\hline
\end{tabular}

Table 2. Numbers of regions

\subsection{Feasible region comparisons}

For completeness, although not directly aligned to MPQP, it is important to compare the feasible regions (or MCAS). Hence Figures 1a,b,c overlay the MCAS for MPC (solid line) with RMPC (shaded) for $n_{c}=0,2,5$ respectively. Unsurprisingly, the robust algorithm has more restricted 

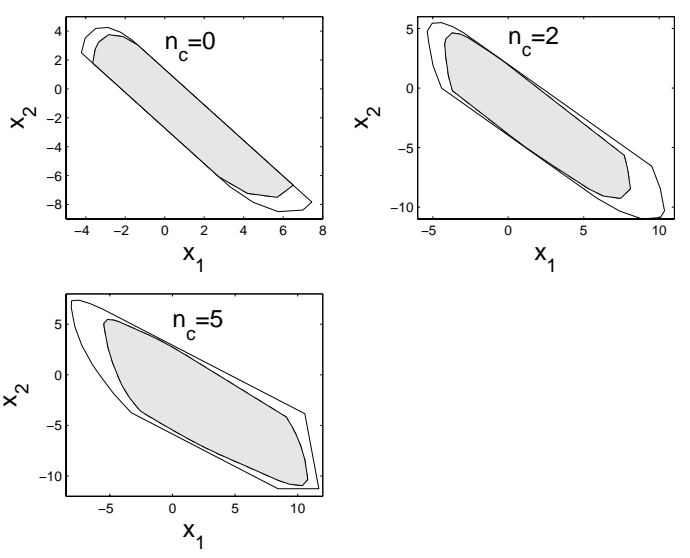

Fig. 1. Feasible regions with $n_{c}=0,2,5$.
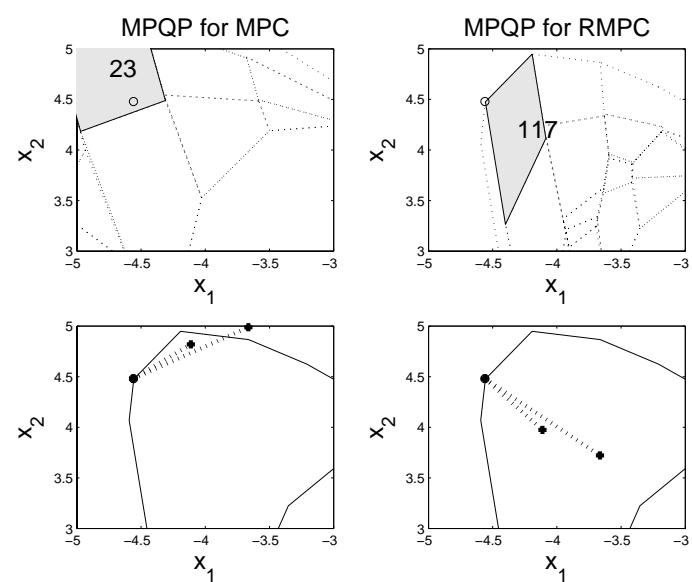

Fig. 2. MPQP regions and closed-loop predictions.

feasibility to account for all possible state evolutions, or in other words the nominal algorithm is in danger of suggesting solutions which lead to infeasibility (are outside $S_{r}$ ) and hence could fail.

4.3.1. Behaviour comparisons Next, we explore in more detail how the control strategy changes for a given region. To this end, we display in figure $2 \mathrm{a}, \mathrm{b}$ and for $n_{c}=3$, the MPQP regions for a specific part of the state space. The state $x=[-4.56,4.48]^{T}$ (marked with a 'o') is in region 117 for RMPC and region 23 for MPC (regions are shaded). The corresponding control laws use:

$$
\begin{array}{l|l}
R M P C & c_{0}=\left[\begin{array}{ll}
1.35 & -0.319
\end{array}\right] x+6.66 \\
M P C & c_{0}=\left[\begin{array}{ll}
0.146 & -0.114
\end{array}\right] x+1.09
\end{array}
$$

which are clearly very different. We then overlay on the figure $2 \mathrm{c}, \mathrm{d}$ the cone of one step ahead trajectories (marked by a dashed line and ' + ') that would arise for one-step ahead prediction. It is clear that these are very different for the two control laws with the MPC predictions (figure 2c) exiting the feasible region $S_{r}$ whereas the RMPC predictions (figure $2 \mathrm{~d}$ ) remain inside and are obviously directed towards $S_{v}$.

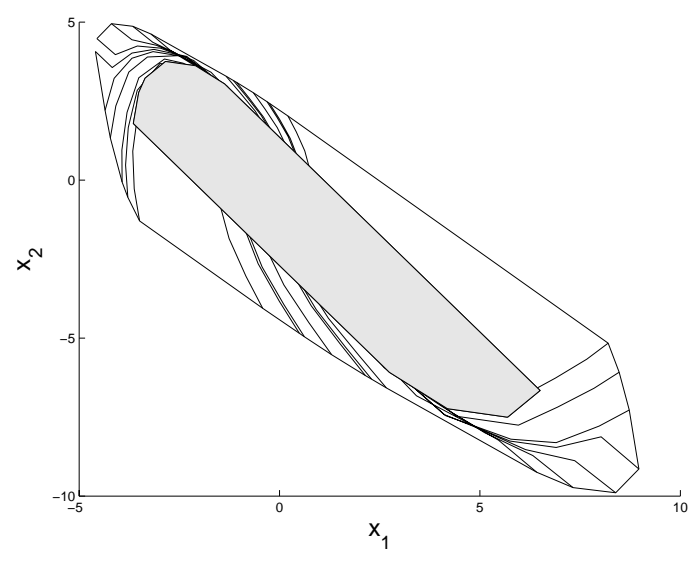

Fig. 3. RMPC closed-loop trajectories.

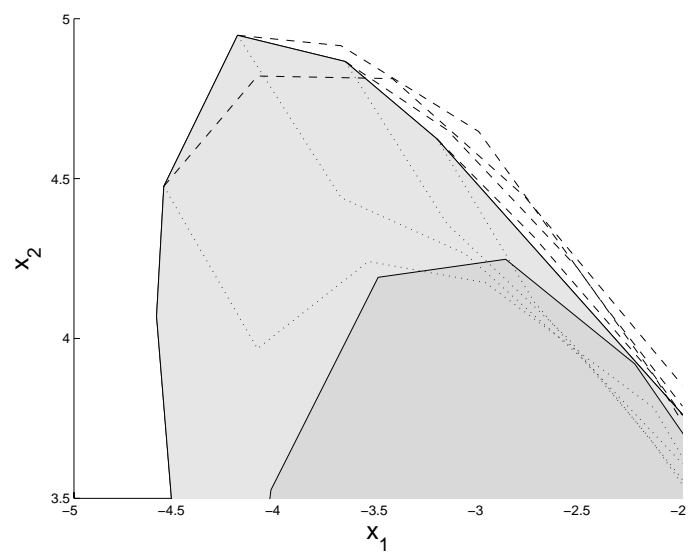

Fig. 4. RMPC and MPC closed-loop trajectories.

\subsection{Closed-loop simulations}

Finally, for completeness, we illustrate the behaviour of the MPQP solution to RMPC over the whole state space. Figure 3 presents the closedloop trajectories for a number of initial conditions on the boundary of $S_{r}(\lambda(k)$ is the same for all these simulations but unknown and time-varying and the shaded area is $S_{v}$ ). What is clear is that all these trajectories remain within the feasible region $S_{r}$.

The corresponding simulations for MPC do not all remain within the feasible region. This is demonstrated in figure 4 (again with $S_{r}, S_{v}$ shaded) where MPC is the dashed lines and RMPC is dotted; clearly the MPC trajectories move in a very different direction and notably some of these exit the feasible region.

\section{CONCLUSIONS}

This paper has demonstrated that MPQP solutions of reasonable complexity can be applied to robust MPC and in particular give a guarantee of convergence and recursive feasibility for a LPV system. We believe this is the first paper giving such results. 
Some points of particular interest are:

(1) MPQP gives a explicit view of how the solution changes to take account of the model uncertainty. Such a view may be very insightful (for instance see figures 2,4).

(2) The single example here seems to show a more rapid increase in the complexity of the MPQP solution with the number of d.o.f. than for the nominal case (table 2). We intend to investigate whether this appears to be generic or specific to the given example.

(3) Robust MPC is often considered an intractable or very complex problem due to the combination of constraint handling with the combinatorial explosion in the number of possible predictions. However, it has been shown here that, in some cases, the MPQP solution is only a little more complex than for an equivalent linear problem and hence is an avenue worth further investigation.

It is also worth reiterating that the results given here are for non-symmetric constraints. Hence the increase in the volume of the feasible region beyond that which ellipsoidal based methods can achieve is very significant.

\section{REFERENCES}

Bemporad. A. and Filippi, C. (2001). Suboptimal explicit MPC via approximate multiparametric quadratic programming. Proc. $C D C$.

Bemporad, A., M. Morari, V. Dua and E.N. Pistokopoulos, 2002, The explicit linear quadractic regulator for constrained systems, Automatica, 38(1), 3-20.

Blanchini, F., 1999, Set invariance in control, Automatica, 35, 1747-1767.

Borrelli, F., Baotic, M., Bemporad, A. and Morari, M., 2001, Efficient on-line computation of constrained optimal control. Proc. IEEE CDC.

Cannon, M., B. Kouvaritakis and J.A. Rossiter, 2001, Efficient active set optimisation in triple mode MPC, IEEE Trans. AC, 46(8), 13071313.

Cannon, M., Deshmukh, V., Lee, Y.I., and B. Kouvaritakis, 2002, Enlargement of polytopic terminal region in NMPC by interpolation and partial invariance, Proc. IEEE CDC.

Gilbert, E.G. and K. T. Tan, 1991, Linear systems with state and control constraints: the theory and application of maximal output admissable sets, IEEE Trans. AC, 36, 9, 1008-1020.

Grieder, P., Borrelli, F., Torrisi, F.D. and Morari, M., 2004. Computation of the constrained infinite time linear quadratic regulator. Automatica, 40(4):701-708.

Imsland, L., Bar, N. and B. Foss, 2004, A new algorithm for efficient MPC and a comparison with competing schemes, Proc. ACC.
Johansen, T.A. and Grancharova, A., 2003, Approximate explicit constrained linear model predictive control via orthogonal search tree. IEEE Trans. AC, 48(5):810-815.

Kerrigan, E., 2000, Robust Constraint Satisfaction: Invariant Sets and Predictive Control, Ph.D Thesis, Cambridge

Kothare, M.V., Balakrishnan, V. and Morari, M., 1996, Robust constrained model predictive control using linear matrix inequalities, $A u$ tomatica, 32, 1361-1379.

Kouvaritakis, B., J.A. Rossiter and J. Schuurmans, 2000, Efficient robust predictive control, IEEE Trans. AC, 45(8), 1545-1549.

Mayne, D.Q., Rawlings, J.B., Rao, C.V. and Scokaert, P.O.M., 2000, Constrained model predictive control: stability and optimality, Automatica, 36, 789-814.

Kvasnica, M., P. Grieder, M. Baotic and M. Morari, 2003, Multi Parametric Toolbox (MPT), http://control.ee.ethz.ch/mpt,

Pluymers, B., Rossiter, J.A., Suykens, J.A.K. and De Moor, B., 2005, The efficient computation of polyhedral invariant sets for linear systems with polytopic uncertainty, ACC

Pluymers, B., Rossiter, J.A., 2005, A simple algorithm for robust MPC, IFAC world congress

Rossiter, J.A., M.J. Rice, and B. Kouvaritakis, A numerically robust state-space approach to stable predictive control strategies. Automatica, 38(1):65-73, 1998.

Rossiter, J.A., 2003, Model based predictive control, CRC press

Rossiter, J.A. and Grieder, P., 2005, Using interpolation to improve efficiency of multiparametric predictive control. Automatica, 41,4.

Schuurmanns, J., and J.A. Rossiter, 2000, Robust piecewise linear control for polytopic systems with input constraints, IEE Proc. CTA, 147, 1, pp13-18

Scokaert, P.O.M. and Rawlings, J.B., 1998, Constrained linear quadratic regulation, IEEE Trans. AC, 43, 8, pp1163-1168.

Tøndel, P., Johansen, T.A. and Bemporad, A., 2003, Evaluation of piecewise affine control via binary search tree. Automatica, 39(5):945-950.

Wan, Z. and M.V. Kothare, 2003, Efficient robust constrained model predictive control with a time-varying terminal constraint set, Systems and Control Letters, 48, 375-383 